I N S T I T U T O

$\mathrm{DE}$

M E D I C I N A

T R O P I C A L

DE

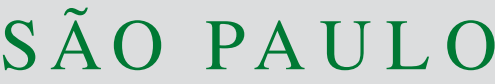

JOURNAL OF THE SÃO PAULO INSTITUTE OF TROPICAL MEDICINE

'Universidade de São Paulo, Faculdade de Medicina, Hospital das Clínicas, São Paulo, São Paulo, Brazil

Universidade de São Paulo, Faculdade de Medicina, Instituto de Medicina Tropical de São Paulo, São Paulo, São Paulo, Brazil

${ }^{3}$ Universidade de São Paulo, Faculdade de Medicina, Hospital das Clínicas, Controle de Infecção, São Paulo, São Paulo, Brazil

Correspondence to: Taniela Marli Bes Universidade de São Paulo, Faculdade de Medicina, Instituto de Medicina Tropical de São Paulo, Av. Dr. Enéas Carvalho de Aguiar, 470, CEP 05403-000, São Paulo, SP, Brazil

Tel: +55 11 3061-7011

E-mail: taniela.bes@usp.br tanielabes@gmail.com

Received: 6 July 2021

Accepted: 23 September 2021

\section{Conjugative transfer of plasmid p_8N_qac(MN687830.1) carrying qacA gene from Staphylococcus aureus to Escherichia coli C600: potential mechanism for spreading chlorhexidine resistance}

\author{
Taniela Marli Bes ${ }^{(1,2}$, Debora Satie Nagano ${ }^{\circledR 2}$, Ana Paula Marchi², \\ Gaspar Camilo², Lauro Vieira Perdigão-Neto', ${ }^{1,2,3}$, Roberta Ruedas Martins ${ }^{2}$, \\ Anna Sara Levin ${ }^{1,2,3}$, Silvia Figueiredo Costa ${ }^{1,2,3}$
}

\section{ABSTRACT}

The methicillin resistant Staphylococcus aureus (MRSA) is recognized by its ability to acquire and transferring resistance genes through interspecies conjugative plasmids. However, transference of plasmids from Gram-positive cocci to Gram-negative bacilli is not well characterized. In this report, we describe the transfer of a conjugative plasmid carrying qacA from MRSA to Escherichia coli $\mathrm{C} 600$. We performed a conjugation experiment using a chlorhexidine resistant MRSA isolate (ST-105/SCCmec type III) carrying the gene qacA and $q a c \mathrm{C}$ as the donor and a chlorhexidine susceptible $E$. coli $\mathrm{C} 600$ isolate as the receptor. Transconjugants were selected using MacConkey agar plates containing chlorhexidine in concentrations ranging from 0.25 to $16 \mathrm{~g}$. $\mathrm{L}^{-1}$. To genotypically confirm the transfer of the resistance gene, the transconjugants were screened by Polymerase Chain Reaction (PCR) and submitted to Sanger's sequencing. MRSA isolates successfully transferred the chlorhexidine resistance gene (qacA) to the recipient $E$. coli strain C600. The E. coli transconjugant exhibited an important reduction of chlorhexidine susceptibility, with MICs increasing from $\leq 0.25$ to $\geq 16$ g. $\mathrm{L}^{-1}$ after conjugation. The qacA gene was detected by PCR as well as in the Sanger's sequencing analysis of DNA from transconjugant plasmids. To the best of our knowledge, this is the first report of the plasmid p_8N_qac(MN687830.1) carrying qacA and its transfer by conjugation from a MRSA to an $E$. coli. These findings increase concerns on the emergence of resistance dissemination across the genus and emphasizes the importance of continuous antiseptic stewardship.

KEYWORDS: Chlorhexidine resistance. Conjugation. Conjugative plasmids. qacA.

\section{INTRODUCTION}

Conjugative plasmids are the main carriers of antibiotic resistance genes. They play a significant role in resistance dissemination and bacterial evolution by accelerating adaptation through horizontal gene transfer, thus providing an opportunity for recombination ${ }^{1}$.

An example of resistance spreading by transfer of mobile genetic elements is methicillin-resistant Staphylococcus aureus (MRSA), a common etiological agent of healthcare associated infections (HAI), with a high antibiotic resistance rate ${ }^{2,3}$. Its ability to acquire and transfer resistance genes and virulence determinants through interspecies conjugative plasmids poses a serious concern for public health. Enterobacteria, such as Escherichia coli, were previously known to acquire mobile 
genetic elements from other genus and are also capable of disseminating these elements containing antimicrobial resistance genes through horizontal transfer ${ }^{4}$. However, studies about spreading of resistance genes from Grampositive to Gram-negative bacteria are limited. HAI among critically ill patients have been shown to increase the risk of morbidity, mortality, as well as the healthcare costs. Some of the approaches to decrease the risk of infection and to prevent resistance dissemination include preoperatory cleansing and daily bathing with chlorhexidine, an ammonium quaternary compound. Bacterial exposure to chlorhexidine results in cellular membrane rupture followed by bacterial death, promoting only minimal skin irritation $^{5}$. Although this strategy is widely used in several countries, its effectiveness remains uncertain and there are concerns if it can promote chlorhexidine resistance ${ }^{6}$. Resistance to ammonium quaternary compounds is typically mediated by three genes $q a c \mathrm{~A}, q a c \mathrm{~B}$ and $q a c \mathrm{C} / s m r$. The gene qac $\mathrm{A}$ encodes an efflux pump that confers export-mediated resistance and its presence was associated with a phenotype of reduced susceptibility towards several biocide compounds ${ }^{7}$. The increased efflux of antibiotics by the outer membrane proteins has gained importance due to their multi-specificity that allows to expel a large number of unrelated substrates ${ }^{8}$.

The emergence of multi-drug resistance by transfer of mobile genetic elements from MRSA to enteric pathogens was proposed as a potential concern by Russell ${ }^{9}$, and now, more than two decades later, we describe the transfer of a conjugative plasmid between a MRSA and an E. coli C600 isolate, the former carrying the qac A gene. This study aimed to report and investigate the role of transferable plasmids between Gram-positive and Gram-negative bacteria.

\section{MATERIALS AND METHODS}

The present study includes an isolate from a previous study conducted by Bes et al. ${ }^{10}$, who identified chlorhexidine resistance among clinical MRSA isolates. Here, a conjugation assay was performed using as the donor an isolate, labeled as $8 \mathrm{~N}$, that had been previously submitted to Whole Genome Sequencing (WGS) by Illumina to characterize its resistance and virulence profile. The $8 \mathrm{~N}$ isolate was identified as a chlorhexidine resistant MRSA, assigned as ST-105, SCCmec III, carrying the genes $q a c \mathrm{~A}$ and $q a c \mathrm{C}$. In addition to chlorhexidine resistance, the isolate carried $\operatorname{erm\mathrm {A}}, \operatorname{erm\mathrm {C}}, m p h \mathrm{C}, m s r \mathrm{~A}, \operatorname{nor} \mathrm{A}$ and $m e c \mathrm{~A}$ conferring resistance to erythromycin, clindamycin, ciprofloxacin and oxacillin ${ }^{10}$. As recipient, we used an Escherichia coli C600 isolate from the American Type Culture Collection (ATCC 23724). E. coli strain C600, primarily described in 1954, is derived from a progenitor strain K-12, first isolated in 1922. It is a laboratory strain that lacks plasmids or restriction enzyme modification systems ${ }^{11}$.

A broth mating experiment was performed among a chlorhexidine resistant MRSA isolate carrying the qacA gene and a chlorhexidine susceptible E. coli C600 isolate, applying an adapted protocol from Ferrières et al. ${ }^{12}$. Donor and recipient cultures had grown overnight at $37^{\circ} \mathrm{C}$ in MacConkey and blood agar medium for the E. coli and for the MRSA isolate, respectively. Up to three colonies of both isolates were transferred individually from the previous culture plate to a fresh $2 \mathrm{~mL}$ of Tryptic Soy Broth (TSB) and were incubated at $37{ }^{\circ} \mathrm{C}$ for approximately $4 \mathrm{~h}$ until cultures reached the OD 600 of 1.2 (corresponding to approximately $10^{9} \mathrm{CFU} / \mathrm{mL}$ ). As controls, recipient and donor isolates were cultured individually and handled similarly to the conjugation assay.

For broth culture mattings, the cultures were mixed at a 1:10 ratio of donor $(100 \mathrm{uL})$ and recipient $(1,000 \mathrm{uL})$ cells in fresh TSB $(4 \mathrm{ml})$ and incubated at $37{ }^{\circ} \mathrm{C}$ without agitation for $18 \mathrm{~h}, 24 \mathrm{~h}$ and $48 \mathrm{~h}$. For the controls, the TBS fresh solution was added to $1,000 \mathrm{uL}$ of the donor culture and $100 \mathrm{uL}$ of the recipient culture, in addition to $100 \mathrm{uL}$ and 1,000 uL of phosphate-buffered saline (PBS), for the donor and recipient cultures, respectively. During the incubation endpoints, $100 \mathrm{uL}$ of the donor, recipient and control cultures were plated on MacConkey agar plates with chlorhexidine in different concentrations, ranged from 0.25 to $16 \mathrm{~g} \mathrm{~L}^{-1}$, using as reference the agar dilution method ${ }^{13}$ and incubated at $37^{\circ} \mathrm{C}$ for $24 \mathrm{~h}$.

To verify the plasmid transfer, the transconjugants were submitted to genotypic and phenotypic tests. Phenotypically, the expression of the qacA gene was confirmed by comparing susceptibility profiles of controls and transconjugants. The recipient strain E. coli C600 was susceptible to chlorhexidine prior to conjugation $(\mathrm{MIC}<0.25)$ and the donor strain MRSA was resistant to chlorhexidine (MIC: 8).

Genotypically, to verify if the plasmid was successfully transferred to the recipient, the transconjugants were harvested from the MacConkey agar plates and the plasmid DNA was extracted using the QIAGEN extraction kit, QIAprep Spin Miniprep (QIAGEN Inc.,Hilden, Germany ). Then, the extracted plasmid DNA was used for the screening of qacA genes by Polymerase Chain Reaction (PCR), using the forward primer 5'-CTATGGCAATAGGAGATATGGTGT and the reverse primer 5'-CCACTACAGATTCTTCAGCTACATG, as previously described ${ }^{14}$. To confirm the outcome, the agarose gel bands were submitted to DNA extraction and sequenced 
by the Sanger's method using MegaBACE 1000 (ABI 3730 DNA Analyser: Applied Biosystems, Thermo Fisher Scientific, Waltham, USA).

\section{RESULTS}

The conjugation assays demonstrated that the chlorhexidine resistance gene from the MRSA (ST-105 $S C C$ mec type III) isolate could be transferred to a recipient E. coli strain C600. The endpoint of $18 \mathrm{~h}$ was selected as it corresponded to the best conjugation in comparison with longer time endpoints. All E. coli transconjugants exhibited significant reductions in the chlorhexidine susceptibility, which raised from a MIC of $\leq 0.25$ to $\geq 16 \mathrm{~g}$. $\mathrm{L}^{-1}$ after conjugation. The experiments, performed in triplicate and in two different trials, presented consistent results, indicating that chlorhexidine resistance genes are capable of conjugal transfer even among different species. The successful plasmid transfer to the recipient was verified by PCR and subsequently by Sanger's sequencing analysis, which confirmed the presence of qacA gene in the transconjugant isolates.

Using Illumina assemblies, it was possible to determine the complete sequence of the plasmid from the transconjugant isolate (GenBank accession number for this nucleotide sequence is MN687830, Supplementary Material). The genome analysis has shown a plasmid sequence with 13,899 base pairs and two origins of replication (rep20 and rep2), named p_8N_qac (Figure 1), which showed high nucleotide identity (97.4\%) with pSAZ_10A(SDOC01000025), a qacA bearing a plasmid from an MRSA ST-239 strain isolated from a wound drainage material of a patient in Malaysia $^{15}$.

\section{DISCUSSION}

In this study, we described a conjugative mobilization of the plasmid p_8N_qac (MN687830.1) carrying the qacA gene from a chlorhexidine resistant MRSA isolate to a chlorhexidine susceptible $E$. coli C600 isolate. To our knowledge, this is the first report highlighting the qac A gene conjugation from a MRSA isolate to a Gram-negative bacillus and the first description of the plasmid p_8N_qac carrying qac A. Previously, qac A has only been found in larger nonconjugative plasmids, such as the ones from the pSKl family, which lack the transfer gene required for conjugation ${ }^{16}$.

Interspecies conjugation was shown by Udo and Grubb $^{16}$, who reported a conjugation experiment with plasmids $p W B G 636(\mathrm{GmR})$ and pWBG642 (EmR) derived from the conjugative plasmid family pWBG637. These plasmids were transferred from $S$. aureus to S. epidermidis and $E$. faecalis strains but were unsuccessful from $S$. aureus to $E$ coli. Then, Charpentier et al. ${ }^{17}$ in 1999 reported the transfer of a conjugative plasmid pIP823 (plasmid family: pUB110/pC194) which contained the $d f r \mathrm{D}$ gene (conferring a high-level of trimethoprim resistance) from Listeria monocytogenes to Gram-negative and Gram-positive bacilli, specifically E. faecalis and E. coli ${ }^{17}$.

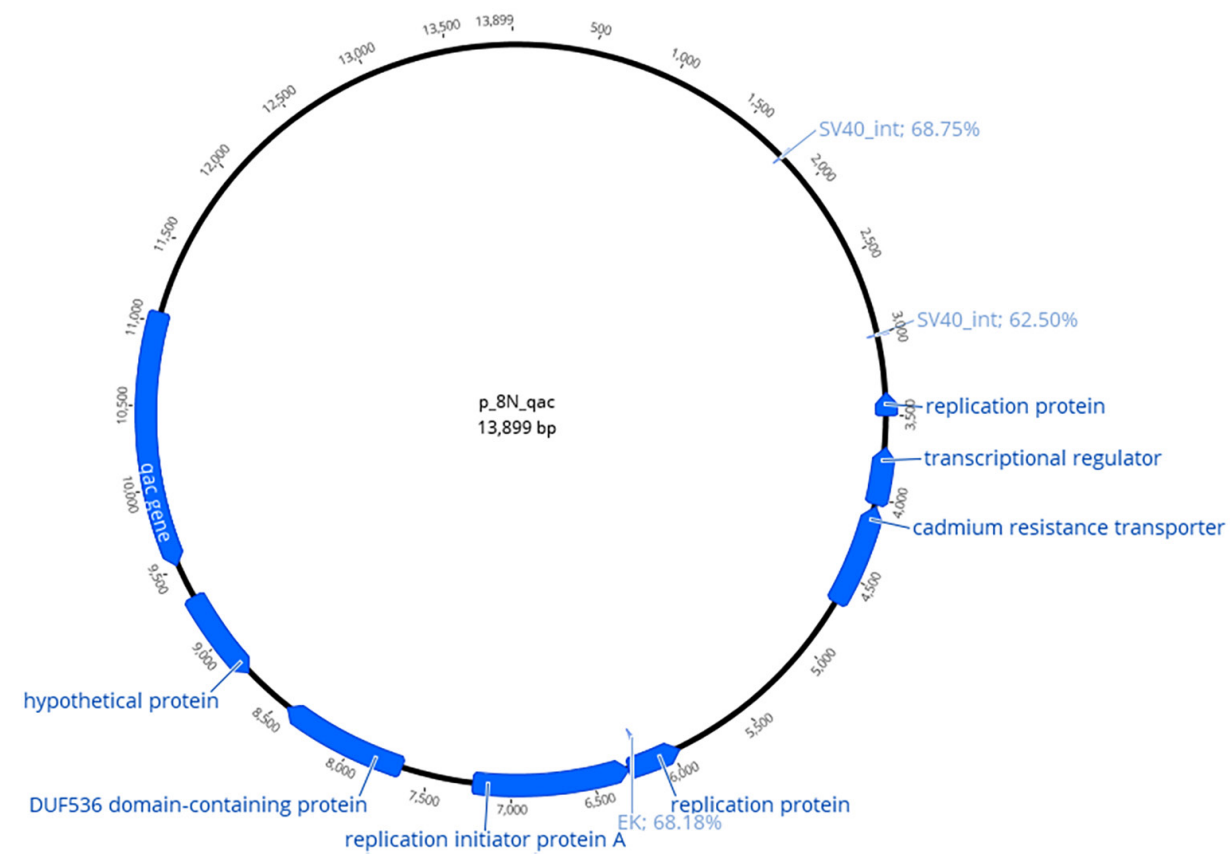

Figure 1 - Simplified pSAZ_10A(SDOC01000025) plasmid map. Regions amplified during the Sanger's sequencing screen are indicated by blue boxes along the plasmid backbone, as well as our gene of interest: qacA. 
In our report, conjugation assays showed that the determinant of chlorhexidine resistance in MRSA isolates can be transferred to the recipient $E$. coli (C600 strain). LaBreck et al. ${ }^{18}$ showed the ability of qacA to be mobilized across Staphylococcus spp. strains, displaying a conjugative transfer of qacA-positive pC02 plasmid (pWBG749family of conjugative plasmids) to several $S$. aureus and $S$. epidermidis isolates among military members from Bethesda, USA, confirmed by WGS. These previous reports make our findings even more thought-provoking, considering that our receptor isolate is a Gram-negative bacillus. Likewise, chlorhexidine is commonly used in preoperatory cleansing and daily bathing as a decolonization strategy to decrease the infection rates by MRSA and other multi-drug resistant Gram-negative bacteria ${ }^{6}$, which are responsible for most of the life threatening infections among severely ill patients in different settings ${ }^{19,20}$.

The hypothesis of horizontal transfer of resistance genes was proposed to explain the spreading of chlorhexidine resistance among enterobacteria, being the qac $\mathrm{A}$ gene transfer through a plasmid-facilitated efflux pump one possibility, which could confer export-mediated resistance across the genus.

\section{CONCLUSION}

Herein we are reporting the first evidence of conjugative transfer of qacA from a Gram-positive coccus to a Gram-negative bacillus, in addition to indicating that the conjugative transfer is sufficient to decrease the susceptibility to chlorhexidine among recipient isolates. Although the present experiment does not allow us to confirm that chlorhexidine resistance is caused by plasmids dissemination, we are proposing this hypothesis for futures studies and highlighting the importance of continuous antiseptic stewardship.

\section{CONFLICT OF INTERESTS}

None to declare.

\section{FUNDING}

This study was supported by the Medical Investigation Laboratory (LIM-49) of the University of Sao Paulo, Brazil.

\section{REFERENCES}

1. Koonin EV. Horizontal gene transfer: essentiality and evolvability in prokaryotes, and roles in evolutionary transitions. F1000Res. 2016;5: F1000 Faculty Rev-1805.
2. Alibayov B, Baba-Moussa L, Sina H, Zdeňková K, Demnerová K. Staphylococcus aureus mobile genetic elements. Mol Biol Rep. 2014;41:5005-18.

3. Yousefi Nojookambari N, Yazdansetad S, Ardebili A, Saki M, Najjari E. Detection of intercellular adhesion (ica) genes involved in biofilm and slime formation in clinical isolates of Staphylococcus Aureus harboring mecA gene. J Babol Univ Med Sci. 2018;20:27-35.

4. Lundquist PD, Levin BR. Transitory derepression and the maintenance of conjugative plasmids. Genetics. 1986;113:48397.

5. McDonnell G, Russell AD. Antiseptics and disinfectants: activity, action, and resistance. Clin Microbiol Rev. 1999;12:147-79.

6. Pittet D, Angus DC. Daily chlorhexidine bathing for critically ill patients. JAMA. 2015;313:365-6.

7. Costa SS, Ntokou E, Martins A, Viveiros M, Pournaras S, Couto I, et al. Identification of the plasmid-encoded qacA efflux pump gene in meticillin-resistant Staphylococcus aureus (MRSA) strain HPV107, a representative of the MRSA Iberian clone. Int J Antimicrob Agents. 2010;36:557-61.

8. Li XZ, Nikaido H. Efflux-mediated drug resistance in bacteria: an update. Drugs. 2009;69:1555-623.

9. Russell AD. Bacterial resistance to disinfectants: present knowledge and future problems. J Hosp Infect. 1999;43 Suppl:S57-68.

10. Bes TM, Perdigão-Neto L, Martins RR, Heijden I, Trindade P, Camilo G, et al. Susceptibility to chlorhexidine and mupirocin among methicillin-resistant Staphylococcus aureus clinical isolates from a teaching hospital. Rev Inst Med Trop São Paulo 2021;63:e27.

11. Appleyard RK. Segregation of new lysogenic types during growth of a doubly lysogenic strain derived from Escherichia Coli K12. Genetics. 1954;39:440-52.

12. Ferrières L, Hémery G, Nham T, Guérout AM, Mazel D, Beloin C, et al. Silent mischief: bacteriophage Mu insertions contaminate products of Escherichia coli random mutagenesis performed using suicidal transposon delivery plasmids mobilized by broad-host-range RP4 conjugative machinery. J Bacteriol. 2010;192:6418-27.

13. Abuzaid A, Hamouda A, Amyes SG. Klebsiella pneumoniae susceptibility to biocides and its association with $\operatorname{cepA}, \mathrm{qac} \Delta \mathrm{E}$ and qacE efflux pump genes and antibiotic resistance. J Hosp Infect. 2012;81:87-91.

14. Mayer S, Boos M, Beyer A, Fluid AC, Schmitz FJ. Distribution of the antiseptic resistance genes qacA, qacB and qacC in 497 methicillin-resistant and -susceptible European isolates of Staphylococcus aureus. J Antimicrob Chemother. 2001;47:8967.

15. National Center for Biotechnology Information. Staphylococcus aureus strain SAZ_10 plasmid pSAZ_10A, whole genome shotgun sequence. [cited 2021 Sep 23]. 
Available from: https://www.ncbi.nlm.nih.gov/nuccore/ SDOC01000025.1? report=GenBank

16. Udo EE, Grubb WB. Conjugal transfer of plasmid pWBG637 from Staphylococcus aureus to Staphylococcus epidermidis and Streptococcus faecalis. FEMS Microbiol Lett. 1990;60:183-7.

17. Charpentier E, Gerbaud G, Courvalin P. Conjugative mobilization of the rolling-circle plasmid pIP823 from Listeria monocytogenes BM4293 among gram-positive and gram-negative bacteria. J Bacteriol. 1999;181:3368-74.

18. LaBreck PT, Rice GK, Paskey AC, Elassal EM, Cer RZ, Law NN, et al. Conjugative transfer of a novel Staphylococcal plasmid encoding the biocide resistance gene, qacA. Front Microbiol. 2018;9:2664.
19. Amin M, Sirous M, Javaherizadeh H, Motamedifar M, Saki M, Veisi $\mathrm{H}$, et al. Antibiotic resistance pattern and molecular characterization of extended-spectrum $\beta$-lactamase producing enteroaggregative escherichia coli isolates in children from southwest Iran. Infect Drug Resist. 2018;11:1097-104.

20. Abbasi Montazeri E, Khosravi AD, Saki M, Sirous M, Keikhaei B, Seyed-Mohammadi S. Prevalence of extended-spectrum betalactamase-producing enterobacteriaceae causing bloodstream infections in cancer patients from southwest of Iran. Infect Drug Resist. 2020;13:1319-26.

Supplementary Material available from: https://doi.org/10.48331/scielodata.O1A6MB 